\title{
Silk Device for the Treatment of Intracranial Aneurysms, Part 2: Factors Related to Clinical and Angiographic Outcome
}

\author{
Tunca KAYA ${ }^{1}$, Ergun DAGLIOGLU², Erdem GURKAS ${ }^{3}$, Ilkay AKMANGIT ${ }^{1,4}$, Ahmet PEKER $^{4}$, Deniz BELEN ${ }^{2}$, \\ Dogan DEDE ${ }^{1}$, Atilla Halil ELHAN ${ }^{5}$, Anil ARAT ${ }^{1,4}$
}

\begin{abstract}
${ }^{1}$ Ankara Numune Training and Research Hospital, Department of Radiology, Ankara, Turkey
${ }^{2}$ Ankara Numune Training and Research Hospital, Department of Neurosurgery, Ankara, Turkey

${ }^{3}$ Ankara Numune Training and Research Hospital, Department of Neurology, Ankara, Turkey

${ }^{4}$ Hacettepe University, School of Medicine, Department of Radiology, Ankara, Turkey

${ }^{5}$ Ankara University, School of Medicine, Department of Biostatistics, Ankara, Turkey
\end{abstract}

\section{ABSTRACT}

AIM: To report patient and procedure-related factors affecting the angiographic and clinical outcome in patients treated with the Silk device.

MATERIAL and METHODS: All patients with intracranial aneurysms in whom treatment was attempted with the Silk flow diverter by our neurovascular team between October 2010 and November 2013 were included consecutively. The data was analyzed by an independent stroke neurologist not involved in the treatment of the patients.

RESULTS: A total of 96 patients (64 female) with ages range from 3 to 78 were included in this study. We found that 54 of the patients were asymptomatic and 42 of them symptomatic, while 21 had a prior history of subarachnoid hemorrhage (SAH). Mean aneurysm size was $10.2 \mathrm{~mm}$ (range 2 to $40 \mathrm{~mm}$ ). 2 patients died due to consequences of SAH. 3 patients developed visual decline on the follow-up, 2 of these were procedure-related. Symptomatic thromboembolic events were noted in 7 cases. Patients with aneurysms smaller than $13 \mathrm{~mm}$ had significantly less complications and higher occlusion rates. The complication rate was significantly high in patients admitted with symptoms. Adjunctive coiling had no impact on outcome.

CONCLUSION: Safety and efficacy of flow diversion in this series was closely related to aneurysm size and presenting symptoms. A size cut-off for safety and efficacy has not been reported before and will be useful not only for future studies but also for patient counseling in daily practice. The futility of adjunctive coiling in this series calls for reappraisal of the current recommendations for this specific device.

KEYWORDS: Silk device, Intracranial aneurysms, Outcome

\section{INTRODUCTION}

Flow diverter (FD) devices have been the current endovascular treatment option for the hardly treated intracranial widenecked, fusiform and giant aneurysms (7). Recently, flow diverter stents have been the most important treatment modality because of acceptable morbidity, mortality and high aneurysm occlusion rates (5). Except for a few small studies, experimental studies in the literature consist of studies where other adjunct devices other than flow diverters are not used and they do not represent the small narrownecked aneurysms frequently seen in routine daily practice. In these studies, flow diverter stents direct the blood flow along the parent artery (13) and provide stasis of the blood inside the aneurysm to promote thrombosis of the aneurysm 
and also function as a scaffold for the neo-intimal growth of the parent artery (6). In clinical studies, FD devices were used alone without using adjunct devices in the literature. Although there is manufacturer disclosure regarding the use of these devices with coils for aneurysms larger than $15 \mathrm{~mm}$, there is no literature data present for the concomitant use of coils and other devices with FD stents.

FD stents were usually used for the unruptured and internal carotid artery located aneurysms, but nowadays they are frequently used for aneurysms with different locations, although number of published reports for their use in other sites are relatively small (19).

Additionally, all published and shared experiences in the literature emphasize the functionality and efficiency of FDs. This is well known and experienced by many operators and clinics (18) but surprisingly the factors associated with aneurysm occlusion rates and variables playing a role in complication formation have not been considered, and the comparative role of adjunct endovascular techniques, aneurysm or patient characteristics on the outcome have not been studied. In this study, our aim was to discuss the above previously unmentioned issues in a series of patients with intracranial aneurysms treated with the Silk Flow Diverter Device (Balt Extrusion, Montmorency, France).

\section{MATERIAL and METHODS}

All the patients with intracranial aneurysms treated with the Silk device (Balt Extrusion, Montromercy, France) by our neurovascular team between October 2010 and November 2013 were included consecutively regardless of the success of the procedure. The data were analyzed by an independent stroke neurologist not participated to the procedures. Treatment and characteristics of the aneurysms were evaluated by the same neurologist. For the final evaluation, only the patients with a clinical and/or angiographic follow-up were included. Early outcomes of the patients treated were discussed in detail in the first part of the study.

Patients were called for the clinical and non-invasive radiological (magnetic resonance imaging or computed tomography) follow-up in between 1-3 months. In addition all the patients were recalled for the digital subtraction angiography at 6 months. Then periodical follow-up was continued as once a year.

Aneurysm occlusion rate and parent artery patency were measured using the Byrne Axis 1 and Axis 2 classification (2).

106 patients with 127 aneurysms were treated in 116 procedures using the Silk device. Among these patients, only the patients with clinical and radiological follow-up were included in the final study group. (96 patients, 113 aneurysms and 104 procedures). Statistical analysis was performed for 104 procedures. Statistical analysis regarding angiographic outcome was made only for the patients with imaging followup and statistical analysis regarding clinical results regardless of the procedural success was made for the patients with clinical follow-up.

\section{Statistical Analysis}

Categorical variables were assessed by Chi-square test or Fisher's Exact test, where applicable. The Shapiro-Wilk test was used to test normality assumptions of the continuous variables. Continuous variables were evaluated by Student's $t$ test or Mann-Whitney $U$ test, where appropriate. A multiple logistic regression was performed to identify the independent risk factors of outcome variables and adjusted odds ratios with their 95\% confidence intervals were calculated. Receiver operating characteristic (ROC) curves were used to describe the performance of diagnostic value of alternative tests. A p value less than 0.05 was considered as significant.

\section{RESULTS}

A total of 96 patients (32 [33.3\%] male, 64 [66.7\%] female) with an age range of 3 to 78 years were included in this study. We found that $54(56.3 \%)$ of the total number of patients were asymptomatic and $42(43.7 \%)$ of them were symptomatic, while $21(21.8 \%)$ had subarachnoid hemorrhage (SAH) in their history. Signs and symptoms at admission were $\mathrm{SAH}$ in $26(27.8 \%)$ patients, headache in $16(16.7 \%)$ patients, recanalization in $10(10.41 \%)$ patients, diplopia in 1 (1.04\%) patient and third nerve palsy in $1(1.04 \%)$ patient, left upper extremity hypoesthesia in $1(1.04 \%)$ patient, ophthalmoplegia in $1(1.04 \%)$ patient. The aneurysms in $40(41.7 \%)$ patients were found incidentally. Aneurysm size ranges from 2 to 40 $\mathrm{mm}$ with an average diameter of $10.17 \mathrm{~mm}$.

Locations of the 113 aneurysms are shown in Table I.

112 of the 116 procedures (96.5\%) were completed with a successful stent placement. Among 4 unsuccessful procedures where stents were malpositioned, stent catheter was prolapsed inside the aneurysm while navigating the stent

Table I: Localizations of Aneurysms

\begin{tabular}{ll}
\hline Total number of aneurysms & 113 \\
\hline Anterior circulation & $97(85.8 \%)$ \\
\hline Posterior circulation & $16(14.1 \%)$ \\
\hline Supraclinoid & $41(36.28)$ \\
\hline Paraophthalmic & $23(20.35 \%)$ \\
\hline Middle cerebral artery & $17(15.04 \%)$ \\
\hline Petrocavernous & $12(10.61 \%)$ \\
\hline Vertebral artery V4 segment & $7(6.19 \%)$ \\
\hline Vertebrobasilar junction & $3(2.65 \%)$ \\
\hline Mid-basilar segment & $3(2.65 \%)$ \\
\hline Anterior communicating artery & $3(2.65 \%)$ \\
\hline Basilar tip & $1(0.88 \%)$ \\
\hline Cervical segment of the internal carotid & $1(0.88 \%)$ \\
\hline artery &
\end{tabular}


several times in the first patient, and the catheter and device were therefore retrieved without flow diversion. In the second patient, the microcatheter could not be navigated across the aneurysm neck. In the third patient, the stent was retrieved because of the malposition of the stent. In the last patient, the stent could not be placed due to the severe spasm in the proximal portion of the parent artery.

In one patient, the scaffolding Leo stents (Balt Extrusion, Montmorency, France) were seen to occlude the aneurysm totally as the Silk device was navigated, the device was thus retrieved and procedure was completed without using Silk device.

In 32 (42.3\%) out of 104 procedures, stents were used, in 26 (25\%) out of 104 procedures, coils were used as an adjunct to the Silk device. In 13 patients, both coils and stents were used simultaneously with Silk device. In addition 12 (37.5\%) stents had been placed in the preceding treatment sessions.

2 patients died due to effects of the SAH. 3 patients developed visual decline in the follow-up, 2 of them are thought to be due to flow diversion procedure. In 7 patients, a thromboembolic event developed. In one of these patients, it was due to the premature discontinuation of the antiplatelet treatment, which resolved without sequela. In another patient, on follow-up, thromboembolic event occurred related to the supplied territory of the artery jailed by the flow diverter. The patient was unable to provide a reliable history of adherence to clopidogrel. This patient had unilateral lower extremity severe paresis. In one patient, there was total occlusion of the ipsilateral middle cerebral artery (MCA) during the preprocedural angiogram which was recanalized partially with a stentriever; the aneurysm was treated successfully but the patient had severe hemiparesis on follow-up. In the 3 patients with total occlusion, 1 patient died due to cerebral infarction, the other 2 patients died due to hemorrhagic conversion of the cerebral infarctions. 1 patient was discharged to home with neurologic deficit but was later lost to follow up and not included in the final analysis. Intracerebral hemorrhage was observed in 2 patients, both resulting with exitus, one was on the contralateral side and the other was due to arterial rupture while inflating the balloon for the FD stent. 1 patient died due to myocardial infarction on follow-up. One patient had ataxia because of instent re-stenosis. In a patient with giant aneurysm, patient was decided to be intubated during the post-procedural first 48 hours for a better control of the arterial blood pressure and bleeding, but during the intensive care unit stay the patient had hypoxic brain injury resulting in death because of accidental extubation. In 1 patient, multiple Silk devices were placed for a dissecting aneurysm in the basilar artery, the patient had contralateral hemiparesis on follow-up.
1 patient had self-limited carotid-cavernous fistula (CCF) during the procedure and CCF remained asymptomatic during follow-up. Procedure-related mortality and severe morbidity (mrs $\geq 3$ ) was found as $9.6 \%$.

96 procedures with imaging follow-up available were classified according to the Byrne Axis 1 (degree of aneurysm occlusion). On control angiograms, Grade 4 (complete occlusion) was observed in 76 (79.1\%) aneurysms, Grade 3 (Contrast filling limited to aneurysmal neck) was seen in 17 (17.7\%) aneurysms, Grade 2 (Less than $50 \%$ contrast filling of the aneurysmal sac) was seen in 3 (3.12\%) aneurysms and Grade 1 (More than $50 \%$ contrast filling of the aneurysmal sac) was seen in 1 (1.05\%) patient, no aneurysm was grade 0 (no change in the endoaneurysmal flow).

For parent artery patency, Byrne Axis II (patency status of the parent artery) classification was used. For the 96 procedures with radiological follow-up, $89(92.7 \%)$ were grade a (no change in parent artery), $3(3.12 \%)$ arteries were grade b (parent artery stenosis) and $4(4.16 \%)$ were grade c (parent artery occlusion).

Likelihood of having unfavorable outcome for patients whose aneurysms were greater than $13 \mathrm{~mm}$ was 9.63 times higher than that of patients whose aneurysms were smaller. $(p=0.004)(95 \% \mathrm{Cl}$ for Adjusted OR 2.10-44.26) According to ROC analysis, cut-off value of 13 was selected for aneurysm size with a sensitivity of $70 \%$ and specificity of $79.8 \%$ to discriminate patients with and without complications (Area under the ROC curve \pm Standard Error $(A \cup C \pm S E)=0.762 \pm 0.082$, $95 \% \mathrm{Cl}$ for AUC 0.600-0.923, $\mathrm{p}=0.007$ ). Likelihood of having complication for patients who admitted to the hospital with symptoms was 6.51 times higher than that of patients who were asymptomatic. $(p=0.033)(95 \% \mathrm{Cl}$ for Adj OR1.16-36.50) (Table II).

Additionally, for the 26 (25\%) out of 104 procedures the use of coils as adjunct to Silk device had no effect either on complication rate $(p=0.116)$ or on aneurysm occlusion rate $(p=0.774)$.

Likelihood of total aneurysm occlusion for patients whose aneurysm size is less than $13 \mathrm{~mm}$ is 3.15 times higher than that of patients with larger aneurysms. $(p=0.040)(95 \% \mathrm{Cl}$ for Adjusted OR 1.05-9.44).

Likelihood of parent artery occlusion or narrowing for patients who were admitted to the hospital with symptoms was 10.28 times higher than that of patients who were asymptomatic. $(p=0.036)(95 \% \mathrm{Cl}$ for Adj OR 1.17-90.55). There was no difference in the rate of adverse events when procedures involving the anterior and posterior circulations were compared (Chi Square test, $p=0.39$ ).

Table II: Risk Factors for Complications, Adjusted for Age and Sex

\begin{tabular}{lccccc}
\hline Risk Factors for Complications & OR & 95\% Cl for OR & OR Adj & 95\% Cl for OR adj & p value \\
\hline Aneurysm size $\geq 13 \mathrm{~mm}$ & 9.21 & $2.18-39.00$ & 9.63 & $2.1-44.26$ & 0.004 \\
\hline Symptomatic & 6.16 & $1.24-30.64$ & 6.51 & $1.16-36.50$ & 0.033 \\
\hline
\end{tabular}




\section{DISCUSSION}

Operator experience and widespread use have been increasing for the FD stents, which as a result widens the therapeutic spectrum. Mortality and morbidity rates regarding the use of FD stents especially for ICA aneurysms are well known, the rates of complications and aneurysm occlusions in this localization are reported in the literature (1). Although these devices have become widely used, surprisingly the factors contributing to complication formation and aneurysm occlusion have not been determined and presented enough. Notwithstanding the fact that the previous studies on FD have generally focused on the implementation of the devices alone (without the use of coils or stents) and mostly in patients without acute presentation (such as subarachnoid hemorrhage or acute infarction), in most practices, use of adjunctive devices in a variety of clinical presentations is the reality. We tried to determine the factors affecting the final outcome in EVT with flow diverters.

In this series, 10 (9.6\%) out of 104 procedures resulted in procedure related mortality or significant morbidity. In the literature, the rate of mortality and morbidity changes from $0 \%$ to $22 \%(1,16)$.

\section{Factors Affecting Clinical Outcome}

In our series, the most important variable affecting the outcome was the size of the treated aneurysm. The risk for complication occurrence decreased significantly as the aneurysm size decreased. For the aneurysms with a size of less than $13 \mathrm{~mm}$, the rate for an unfavorable outcome at a mean follow-up of 8 months was $2.63 \%$ and is comparable to that of coiling with or without balloon/stent assistance $(12,15)$.

As the size of the majority of the treated aneurysms is less than $10 \mathrm{~mm}$, FD stents have become an alternative to the surgical or conventional endovascular techniques for the treatment of small intracranial side wall or fusiform aneurysms.

For a final conclusion, randomized comparison of FD's and conventional treatment modalities is needed for the treatment of such aneurysms.

The second factor contributing to unfavorable outcome is the presence of symptoms at admission. We found significantly low unfavorable outcome for the incidentally found aneurysms treated with FD stents (Adj. OR 6.51). This finding directs us to use the conventional treatment strategies in the acute setting for the patients presenting with $\mathrm{SAH}$ to prevent re-rupture and to use FD stents afterwards in another treatment session when the patient is clinically stable.

In our series, FD use in perforator rich arteries when compared to FD use at locations where no perforating branches exist do not exert any significance in terms of complications. This finding suggests that FD use is safe in perforator rich arteries. But in perforator rich arteries, use of multiple FDs results in increased risk of procedure related permanent complications. As strut concentration increases significantly with simultaneous use of multiple FDs, this can cause the occlusion of the perforating branches with a resultant significant increase of the complication rates. On the other hand, concomitant use of stent with FDs in perforator rich arteries did not increase the procedure related complication rates. Coil use was not associated with additional morbidity and mortality on follow-up. Results were also confirmed with size-adjusted analysis.

\section{Factors Affecting Angiographic Results on Follow-Up}

As consistent with the literature, total occlusion rate was succeeded in $76(79.1 \%)$ of the procedures $(5,12)$. Aneurysm size was also a statistical variable for aneurysm occlusion rates as in the case of clinical outcome. This suggests that the high occlusion rates of larger aneurysms encountered in the early trials including selected cases $(8,10,11)$ may not be observed in real world applications. This phenomenon of discordant study and real-world results has been observed in the cardiology literature previously (4).

Another interesting result was the demonstration of the absence of a favorable effect of intrasaccular coils on the final angiographic result. This finding was reproduced in sizeadjusted analysis as well.

The status of the parent artery during follow-up was influenced by the symptoms of the patients on admission to the hospital. Again in early trials, patients presenting with $\mathrm{SAH}$ or dissection at acute stage were very limited in number although in the real world experience FDs can be used for these patients. In our series, parent artery compromise was seen more frequently. Among our patients, 4 (4.6\%) have developed parent artery occlusion but the parent artery occlusion has been reported up to $14 \%$ of aneurysms in the literature (2). It is possible that our vigorous anti-aggregation regimen may have led to a decrease of parent artery occlusions at expense of a better occlusion rate in our cohort.

The patency of the parent artery was not related to the diameter of the device used for treatment in our series suggesting that multiple patient related and extrinsic factors may play a role in this phenomenon.

In our cohort, we observed fish mouthing (distal tapering) as reported in recent series, but unlike these recent reports, we did not observe creeping stent phenomenon. The rate of these two phenomena were reported as $13 \%$. In our series, configuration change was $1 \%$ which is remarkably low when compared to studies where the same stent was used (3).

In our series, for large aneurysms, aneurysm filling was more frequently observed at angiographic follow-up. We have also seen aneurysms with ceased angiographic filling although the size of these aneurysms did not diminish or even increased on sectional imaging. We have also found aneurysms with no angiographical filling that increased in size on sectional imaging. This condition was not classified in the current angiographic follow-up classification. In addition, recently as also seen in our series, when used in the treatment of either bifurcation aneurysms or side wall aneurysms close to bifurcations, FDs are elongated covering the bifurcations and the branches. But when FDs are positioned including bifurcations, development of stenosis or occlusion on jailed branches are encountered more frequently than on parent arteries (14). Similarly, this is not present in the current outcome classification. 


\section{CONCLUSION}

Flow diversion appears to be safer and more effective in small aneurysms. As such, this treatment can potentially be put forward as standard therapy for aneurysms amenable for treatment with this method in both anterior and posterior circulations. For larger aneurysms, the efficacy of flow diverters decrease but it is still comparatively high. A higher adverse event rate should be kept in mind especially in symptomatic patients with large aneurysms. The current recommendation of adjunctive coiling in patients with aneurysms of $15 \mathrm{~mm}$ or more in size was not supported by our findings.

\section{- REFERENCES}

1. Briganti F, Napoli M, Tortora F, Solari D, Bergui M, Boccardi E, Cagliari E, Castellan L, Causin F, Ciceri E, Cirillo L, De Blasi R, Delehaye L, Di Paola F, Fontana A, Gasparotti R, Guidetti G, Divenuto I, lannucci G, Isalberti M, Leonardi M, Lupo F, Mangiafico S, Manto A, Menozzi R, Muto M, Nuzzi NP, Papa R, Petralia B, Piano M, Resta M, Padolecchia R, Saletti A, Sirabella G, Bolgè LP: Italian multicenter experience with flow-diverter devices for intracranial unruptured aneurysm treatment with periprocedural complications-a retrospective data analysis. Neuroradiology 54(10):1145-1152, 2012

2. Byrne JV, Beltechi R, Yarnold JA, Birks J, Kamran M: Early experience in the treatment of intra-cranial aneurysms by endovascular flow diversion: A multicentre prospective study. PLoS One 5(9):pii: e12492,2010

3. Cohen JE, Gomori JM, Moscovici S, Leker RR, Itshayek E: Delayed complications after flow-diverter stenting: Reactive in-stent stenosis and creeping stents. J Clin Neurosci 21(7):1116-1122, 2014

4. Colombo A, Chieffo A: Drug-eluting stent update 2007: Part III: Technique and unapproved/unsettled indications (left main, bifurcations, chronic total occlusions, small vessels and long lesions, saphenous vein grafts, acute myocardial infarctions, and multivessel disease). Circulation 116(12):1424-1432, 2007

5. D'Urso PI, Lanzino G, Cloft HJ, Kallmes DF: Flow diversion for intracranial aneurysms: A review. Stroke 42(8):2363-2368, 2011

6. Fiorella D, Lylyk P, Szikora I, Kelly ME, Albuquerque FC, McDougall CG, Nelson PK: Curative cerebrovascular reconstruction with the Pipeline embolization device: The emergence of definitive endovascular therapy for intracranial aneurysms. J Neurolntervent Surg 1(1):56-65, 2009

7. Fiorella D, Woo HH, Albuquerque FC, Nelson PK: Definitive reconstruction of circumferential, fusiform intracranial aneurysms with the pipeline embolization device. Neurosurgery 62:1115-1120, 2008
8. Kallmes DF, Hanel R, Lopes D, Boccardi E, Bonafé A, Cekirge $S$, Fiorella D, Jabbour P, Levy E, McDougall C, Siddiqui A, Szikora I, Woo H, Albuquerque F, Bozorgchami H, Dashti SR, Delgado Almandoz JE, Kelly ME, Turner R 4th, Woodward BK, Brinjikji W, Lanzino G, Lylyk P: International retrospective study of the pipeline embolization device: A multicenter aneurysm treatment study. AJNR Am J Neuroradiol 36(1):108115, 2015

9. Kamran M, Yarnold J, Grunwald IQ, Byrne JV: Assessment of angiographic outcomes after flow diversion treatment of intracranial aneurysms: A new grading schema. Neuroradiology 53(7):501-508, 2011

10. Lylyk P, Miranda C, Ceratto R, Ferrario A, Scrivano E, Luna $H R$, Berez AL, Tran Q, Nelson PK, Fiorella D: Curative endovascular reconstruction of cerebral aneurysms with the pipeline embolization device: The Buenos Aires experience. Neurosurgery 64(4):632-642, 2009

11. Nelson PK, Lylyk P, Szikora I, Wetzel SG, Wanke I, Fiorella D: The pipeline embolization device for the intracranial treatment of aneurysms trial. AJNR Am J Neuroradiol 32(1):34-40, 2011

12. Piotin M, Blanc R: Balloons and stents in the endovascular treatment of cerebral aneurysms: Vascular anatomy remodeled. Front Neurol 5:41, 2014

13. Sadasivan C, Lieber BB, Cesar L, Miskolczi L, Seong J, Wakhloo AK: Angiographic assessment of the performance of flow divertors to treat cerebral aneurysms. Conf Proc IEEE Eng Med Biol Soc 1:3210-3213, 2006

14. Saleme S, Iosif C, Ponomarjova S, Mendes G, Camilleri $Y$, Caire F, Boncoeur MP, Mounayer C: Flow-diverting stents for intracranial bifurcation aneurysm treatment. Neurosurgery 75(6):623-631, 2014

15. Shapiro M, Becske T, Sahlein D, Babb J, Nelson PK: Stentsupported aneurysm coiling: A literature survey of treatment and follow-up. AJNR Am J Neuroradiol 33(1):159-163, 2012

16. Szikora I, Berentei Z, Kulcsar Z, Marosfoi M, Vajda ZS, Lee W, Berez A, Nelson PK: Treatment of intracranial aneurysms by functional reconstruction of the parent artery: The Budapest experience with the pipeline embolization device. AJNR Am J Neuroradiol 31(6):1139-1147, 2010

17. Tähtinen OI, Manninen HI, Vanninen RL, Seppänen J, Niskakangas T, Rinne J, Keski-Nisula L: The silk flow-diverting stent in the endovascular treatment of complex intracranial aneurysms: Technical aspects and midterm results in 24 consecutive patients. Neurosurgery 70(3):617-623, 2012

18. Yu SC, Kwok CK, Cheng PW, Chan KY, Lau SS, Lui WM, Leung KM, Lee R, Cheng HK, Cheung YL, Chan CM, Wong GK, Hui JW, Wong YC, Tan CB, Poon WL, Pang KY, Wong AK, Fung $\mathrm{KH}$ : Intracranial aneurysms: Midterm outcome of pipeline embolization device-a prospective study in 143 patients with 178 aneurysms. Radiology 265(3):893-901, 2012

19. Zanaty M, Chalouhi N, Tjoumakaris SI, Gonzalez LF, Rosenwasser R, Jabbour P: Flow diversion for complex middle cerebral artery aneurysms. Neuroradiology 56(5):381387,2014 